\title{
Hypnotherapy in severe irritable bowel syndrome: further experience
}

\author{
P J WHORWELL, ALISON PRIOR, AND S M COLGAN \\ From the Department of Medicine, University Hospital of South Manchester, Manchester
}

SUMMARY Fifteen patients with severe intractable irritable bowel syndrome previously reported as successfully treated with hypnotherapy, have now been followed up for a mean duration of 18 months. All patients remain in remission although two have experienced a single relapse overcome by an additional session of hypnotherapy. Experience with a further 35 patients is reported giving a total group of 50. This group was divided into classical cases, atypical cases and cases exhibiting significant psychopathology. The response rates were $95 \%, 43 \%$, and $60 \%$ respectively. Patients over the age of 50 years responded very poorly $(25 \%)$ whereas those below the age of 50 with classical irritable bowel syndrome exhibited a $100 \%$ response rate. This study confirms the successful effect of hypnotherapy in a larger series of patients with irritable bowel syndrome and defines some subgroup variations.

In a previous controlled trial we reported that 'gut directed' hypnotherapy appeared to be highly effective in the management of patients with severe refractory irritable bowel syndrome.' A criticism of this study has been that the follow up period of three months was too short to discount a placebo response to hypnosis. ${ }^{23}$ All 15 patients treated with hypnotherapy in the original study have now been followed up for over one year and these data are reported here together with experience on an additional 35 patients treated in a similar manner.

\section{Methods \\ PATIENTS \\ The 15 patients receiving hypnotherapy in the original study have been followed up at three monthly intervals with an assessment of symptom scores and an additional session of hypnotherapy at each visit. The mean follow up period was 18 months (14-21 months). Patients were asked to telephone if they experienced a relapse so that an additional session of hypnotherapy could be arranged. A further 35 patients ( 31 women, four men, aged 23-65 years) with intractable irritable bowel syndrome have \\ Address for correspondence: Dr P J Whorwell, Dept of Medicine, University of South Manchester, Nell Lane, West Didsbury, Manchester M20 8LR. Received for publication 11 July 1986.}

also been treated by hypnotherapy and followed for at least three months. These patients were divided into three groups. Group 1: classical cases of irritable bowel syndrome exhibiting abdominal pain, abdominal distension, and an abnormal bowel habit. Group 2: atypical cases lacking one or two of the three criteria necessary for Group 1. Group 3: patients with classical irritable bowel syndrome with significant psychopathology as judged by a score in excess of 14 on the 28 question general health questionnaire. ${ }^{4}$ The original study only included patients in Group 1.

Hypnotherapy was carried out by PJW and consisted of half hour sessions of decreasing frequency over a three month period. Patients were given a tape for daily autohypnosis after the third session. Before hypnosis the patient was given a simple account of intestinal smooth muscle physiology. Hypnosis was induced by an eye fixation and arm levitation technique followed by standard deepening procedures. After general comments about improvement of health and wellbeing, attention was directed to the control of intestinal smooth muscle. The patient was asked to place his/her hand on the abdomen, feel a sense of warmth and relate this to asserting control over gut function. All sessions were concluded with standard ego strengthening suggestions. No subject was unable to be hypnotised. Ten weekly sessions of hypnotherapy were offered before treatment was 
judged as having failed. Patients recorded daily the frequency and severity of abdominal pain and abdominal distension on a scale of $0=$ none, $1=$ mild, $2=$ moderate, or $3=$ severe. Bowel habit was also recorded and abnormality expressed on a similar $0-3$ scale. Overall improvement of symptoms and wellbeing were scored weekly on a $0-3$ scale with 0 indicating no improvement and three maximum improvement. Data for each seven days was totalled to give a weekly score for each parameter.

Patients were judged as having 'improved' only if their symptoms became mild or absent and they required no medication for irritable bowel syndrome with the exception of bulking agents. Two of the nonresponders did not complete diary cards. For the purpose of analysis of results they were assumed to have 'severe' symptoms for each parameter at the beginning and end of the study.

\section{Results}

All of the original group of patients were in remission after a mean follow up period of 18 months (14-21 months) with symptoms not significantly different to those at the end of the previous study. Two patients suffered a serious relapse (one at 12 months, the other at 14 months) which was overcome by an additional session of hypnotherapy.

The overall results for the total group of 50 patients are shown in Tables 1 and 2 . The overall success rate was $84 \%$ but it is apparent that classical cases respond best $(95 \%)$. Although the numbers are small, patients with atypical symptoms appear to do badly whatever their age $(43 \%)$ and classical cases and patients with psychological problems are less likely to

Table 1 Results for all 50 patients treated

\begin{tabular}{lrcl}
\hline & $(n)$ & Improved & Not improved \\
\hline Classical cases (group 1) & 38 & $36(95 \%$ & 2 \\
Atypical cases (group 2) & 7 & $3(43 \%)$ & 4 \\
Classical cases with high & 5 & $3(60 \%)$ & 2 \\
GHQ* (group 3) & 50 & $42(84 \%)$ & $8(16 \%)$ \\
Total & 50 & & \\
\hline
\end{tabular}

${ }^{*} \mathrm{GHQ}=$ general health questionnaire.

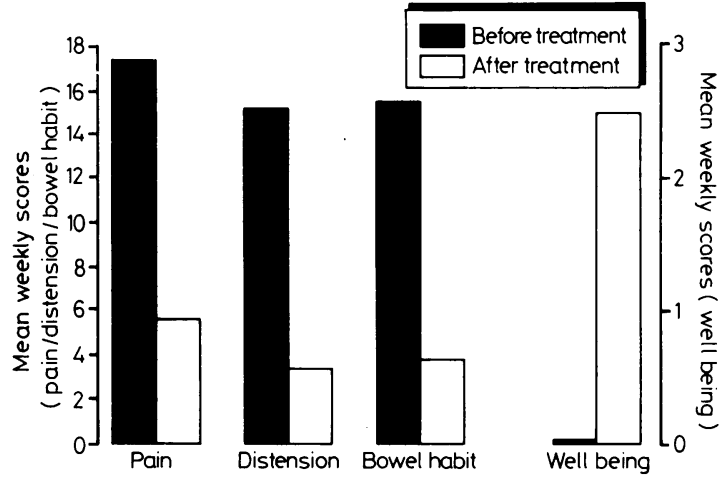

Figure Change in individual symptoms with treatment.

respond over the age of 50 years. All non-responders were women.

The Figure shows the changes in individual symptoms with hypnotherapy. It can be seen that all parameters showed considerable improvement.

\section{Discussion}

This follow up study confirms the longterm efficacy of hypnotherapy in severe irritable bowel syndrome but it is still too early to establish whether the improvement will be permanent. Despite encouragement to continue with regular autohypnosis all patients are still receiving three monthly hypnotherapy because of a clinical impression that this is necessary to maintain remission in some subjects. A controlled study is now in progress to answer this question.

It was felt inappropriate to have a control group for the additional 35 patients reported as our previous controlled trial had shown a clear cut advantage for hypnotherapy. As in the original study the additional patients reported here were all severe cases of irritable bowel syndrome who had proved refractory to conventional forms of therapy and therefore might be expected to have a similar low placebo response.

From the data for the total group of 50 patients it appears that the response to hypnotherapy is dependent on the subject's age. A high success rate was achieved in classical cases (Group 1) under the age of 50 years, which is fortunately the age group

Table 2 Results according to patients age

\begin{tabular}{|c|c|c|c|c|c|c|}
\hline & \multicolumn{3}{|c|}{ Patients under 50 years } & \multicolumn{3}{|c|}{ Patients over 50 years } \\
\hline & $(n)$ & Improved & Not improved & $(n)$ & Improved & Not improved \\
\hline Classical cases & 35 & 35 & 0 & 3 & 1 & 2 \\
\hline Atypical cases & 4 & 2 & 2 & 3 & 1 & 2 \\
\hline Classical cases with high $\mathrm{GHQ}^{*}$ & 3 & 3 & 0 & 2 & 0 & 2 \\
\hline
\end{tabular}

${ }^{*} \mathrm{GHQ}=$ general health questionnaire. 
that accounts for the majority of patients with irritable bowel syndrome. Conversely a poor response rate is emerging in patients over that age.

The atypical cases consisted largely of patients with intractable abdominal pain with little or no abdominal distension or change of bowel habit. Controversy exists as to whether such patients do indeed have irritable bowel syndrome but as long as doubt remains it was felt appropriate to include them within the study in order to assess the role of hypnotherapy in this variant.

The mechanism by which hypnosis exerts its beneficial effect is unclear. We feel that gut directed hypnosis is critically important and this view is supported by the observation that six of the patients reported herein had previously received hypnotherapy of a non-specific nature without any beneficial effect on their irritable bowel syndrome. In order to answer this question conclusively, however, a controlled study is now in progress to compare the effect of hypnotic relaxation with gut directed hypnosis.

In conclusion this additional study confirms the dramatic response of many patients with irritable bowel syndrome to hypnotherapy and helps to define those patients most likely to respond.

SMC is supported by a grant from the North Western Regional Health Authority.

\section{References}

1 Whorwell PJ, Prior A, Faragher EB. Controlled trial of hypnotherapy in the treatment of severe, refractory irritable bowel syndrome. Lancet 1984; 2: 1232-4.

2 Editorial. An irritable mind or an irritable bowel. Lancet 1984; 2: 1249-50.

3 Clare A. Stress management and irritable bowel syndrome. Lancet 1985; 2: 719.

4 Goldberg DP. The detection of psychiatric illness by questionnaire. No 21. Institute of psychiatry. Maudsley Monographs, 1972. 